\title{
Strong-field single-cycle THz pulses generated in an organic crystal
}

\author{
Christoph P. Hauri, ${ }^{1,2, a)}$ Clemens Ruchert, ${ }^{1}$ Carlo Vicario, ${ }^{1}$ and Fernando Ardana ${ }^{1,2}$ \\ ${ }^{1}$ Paul Scherrer Institute, 5232 Villigen, Switzerland \\ ${ }^{2}$ Physics Department, Ecole Polytechnique Federale de Lausanne, 1013 Lausanne, Switzerland
}

(Received 15 July 2011; accepted 21 September 2011; published online 20 October 2011)

\begin{abstract}
We present high-power single-cycle carrier-envelope phase locked $\mathrm{THz}$ pulses at a central frequency of $2.1 \mathrm{THz}$ with $\mathrm{MV} / \mathrm{cm}$ electric field strengths and magnetic field strengths beyond $0.3 \mathrm{~T}$. The $\mathrm{THz}$ radiation is generated by optical rectification in an organic salt crystal 4-N,Ndimethylamino- $4^{\prime}-\mathrm{N}^{\prime}$-methyl stilbazolium tosylate called DAST pumped with the signal wavelength of a powerful optical parametric amplifier. Conversion efficiencies of more than $2 \%$ are reported. (c) 2011 American Institute of Physics. [doi:10.1063/1.3655331]
\end{abstract}

High-power single-cycle $\mathrm{THz}$ pulses are an adequate tool for exploring nonlinear phenomena such as nonlinear carrier transport processes in solids, ${ }^{1,2}$ ultrafast magnetic switching, manipulation of quantum states, ${ }^{3}$ imaging applications, and others. ${ }^{4-7}$ While laser-based $\mathrm{THz}$ radiation at frequencies 20-100 THz has been generated by difference frequency mixing in nonlinear inorganic crystals, ${ }^{8}$ the generation of highpower $\mathrm{THz}$ single-cycle pulses above $1 \mathrm{MV} / \mathrm{cm}$ in the range of $0.1-10 \mathrm{THz}$, the so-called THz gap, is still challenging due to the limited availability of appropriate $\mathrm{THz}$ emitters. ${ }^{9}$ Only recently, single-cycle pulses at $0.8 \mathrm{THz}$ central frequency of 1 $\mathrm{MV} / \mathrm{cm}$ have been demonstrated by optical rectification (OR) in $\mathrm{LiNbO}_{3}$ (Ref. 10) with help of sophisticated pulse front tilting and imaging optics. Other laser-based approaches like plasma-based $\mathrm{THz}$ generation have been used for single-cycle pulse formation up to a few hundreds of $\mathrm{kV} / \mathrm{cm} .{ }^{11}$ Apart from $\mathrm{THz}$ sources based on inorganic crystals, plasma nonlinearities, or large-scale linear accelerator-based schemes, ${ }^{12}$ organic nonlinear crystals have been proposed recently as efficient table-top $\mathrm{THz}$ emitters. ${ }^{13}$ Broadband $\mathrm{THz}$ pulses exceeding $10 \mathrm{THz}$ were recorded for DAST pumped with an $800 \mathrm{~nm}$ Ti:sapphire laser, ${ }^{14}$ and field strength up to $100 \mathrm{kV} /$ $\mathrm{cm}$ at a central frequency of $2 \mathrm{THz}$ could be demonstrated for DAST pumped with a (near-) infrared source. ${ }^{15}$

In this letter, we present high-power single-cycle $\mathrm{THz}$ pulses with electric fields beyond $1 \mathrm{MV} / \mathrm{cm}$ and with a corresponding magnetic transient of larger than $0.3 \mathrm{~T}$. The generation is based on OR in the organic salt crystal $(4-\mathrm{N}, \mathrm{N}-$ dimethylamino- $4^{\prime}-\mathrm{N}^{\prime}$-methyl stilbazolium tosylate) known as DAST which is driven by a powerful femtosecond infrared laser. The nonlinear conversion process gives rise to carrierenvelope phase (CEP) stabilized $\mathrm{THz}$ pulses at a center frequency of $2.1 \mathrm{THz}$ with a bandwidth of $2 \mathrm{THz}$ at full width half maximum (FWHM). High pump-to-THz energy conversion efficiency of up to $2.2 \%$ and high photon conversion efficiency (up to 235\%) are achieved. The setup provides sub-ps $\mathrm{THz}$ transients with a pulse wavefront and transverse beam profile, which allows for tight focusing and the realization of highest fields.

In our experiment (Fig. 1), a $0.2 \mathrm{~mm}$ thick DAST crystal is pumped by an infrared source delivering up to $950 \mu \mathrm{J}$ on target. The infrared source is an optical parametric amplifier

${ }^{a)}$ Electronic mail: christoph.hauri@psi.ch.
(OPA) pumped by a multi-mJ Ti:sapphire laser system running at $100 \mathrm{~Hz}{ }^{16}$ The OPA allows the infrared wavelength to be tuned between 1.2 and $1.5 \mu \mathrm{m}$, which is the relevant spectral range for performing efficient OR in the organic crystal. The pump pulse (Fig. 1(a)) has a duration of 68 fs (FWHM) with the spectral shape shown in Fig. 1(b). For THz generation, the collimated infrared pump beam is sent through the uncoated DAST crystal at normal incidence. A 3-mm thick polytetrafluoroethylene filter is located after the crystal and acts as separator of the transmitted $\mathrm{THz}$, and the residual infrared radiation being scattered. The temporal and spectral $\mathrm{THz}$ pulse characteristics are reconstructed by applying the well-known electro-optical sampling (EOS) technique with a $\mathrm{GaP}$ crystal. ${ }^{17} \mathrm{~A}$ thin crystal $(95 \mu \mathrm{m} \pm 5 \%)$ has been chosen to avoid nonlinear effects in EOS which could potentially be induced by the strong $\mathrm{THz}$ field. In addition, a high-resistivity Si attenuator $(T=65 \%)$ is placed in front of the EO crystal to ensure a linear sampling response. A weak 50 fs pulse acts as a probe $(\lambda=810 \mathrm{~nm})$ and originates from the same Ti:Sapphire amplifier pumping the optical parametric amplifier.

Optical rectification in DAST is enabled by the broad spectrum of the fundamental infrared (Fig. 1(b)). Thanks to $\mathrm{OR}$, the generated $\mathrm{THz}$ electric field is intrinsically CEP stabilized. In fact, the phase fluctuation of the $\mathrm{THz}$ radiation is canceled since OR is equal to intra-pulse difference frequency generation with the two frequencies carrying intrinsically the same CEP offset. ${ }^{18}$ Thus, the CEP offset is inherently locked due to the intrinsic intra-pulse phase coherence of the pump pulse even though neither the infrared pump laser nor the front-end Ti:sapphire laser is CEP stabilized. The intrinsic CEP stabilization of our $\mathrm{THz}$ source offers best conditions for the exploration of field sensitive phenomena since temporal field shapes identical to the one shown in Fig. 2 are provided for consecutive shots.

Compared to inorganic crystals, the DAST provides very large nonlinear optical susceptibility ${ }^{19}$ for optical rectification $\left(\chi_{111}^{(2)}=480 \mathrm{pm} / \mathrm{V}\right.$ at $\left.\lambda_{\mathrm{p}}=1.5 \mu \mathrm{m}\right)$ paired with a low absorption for both the $\mathrm{THz}$ and the pump laser. $\mathrm{THz}$ radiation in DAST is emitted in forward direction collinear with the pump and carries the same linear polarization as the pump pulse. Our simple generation scheme (Fig. 1) allows the formation of well-collimated low-diverging $\mathrm{THz}$ radiation without additional imaging optics since the driving infrared laser pulse transverses the organic crystal in a 

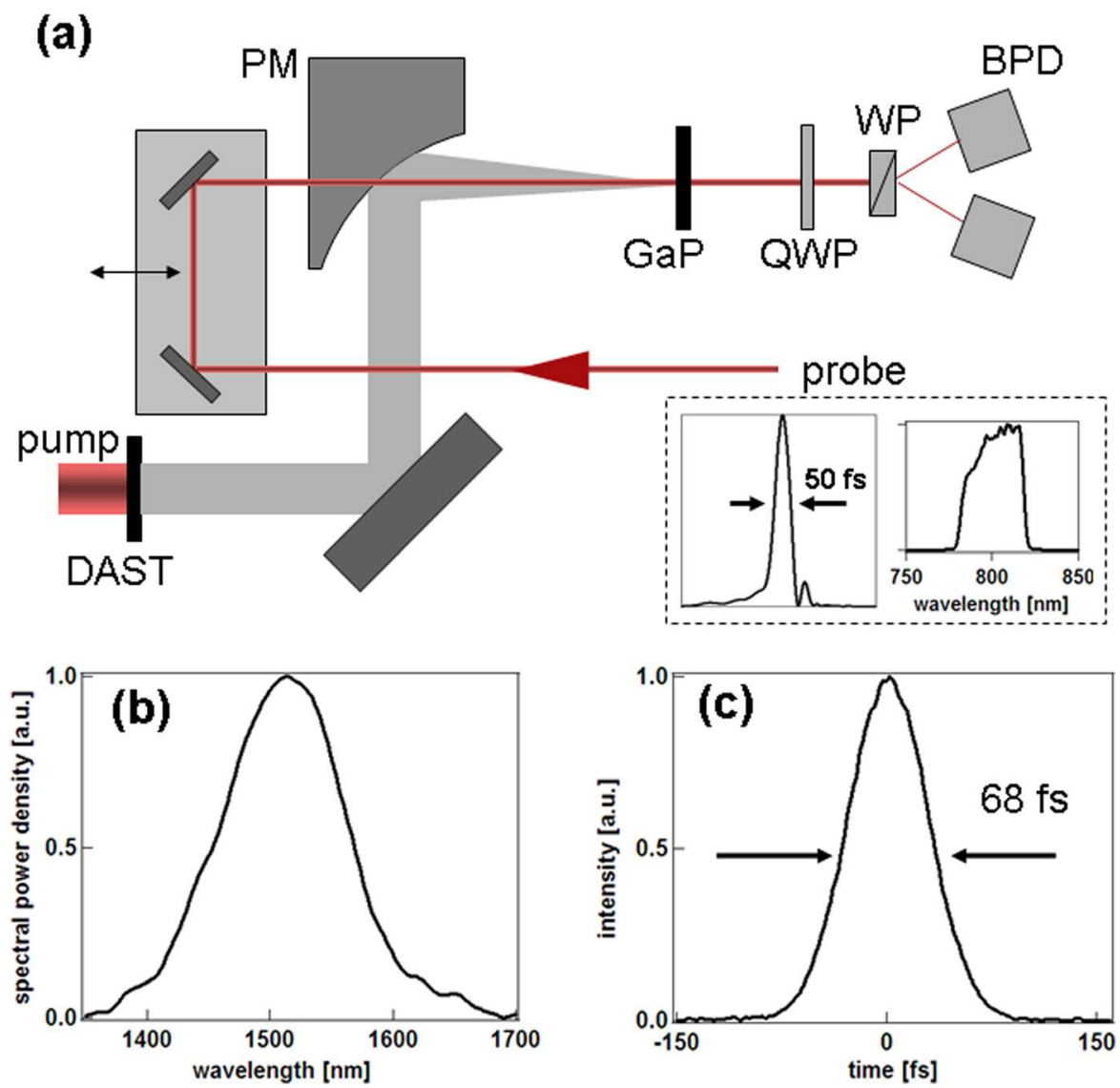

FIG. 1. (Color online) $\mathrm{THz}$ generation with DAST. (a) Schematic setup of generation and electro-optic detection. A 50 fs probe pulse centered at $810 \mathrm{~nm}$ (inset) is used for sampling the THz pulse in GaP sensor $(\approx 95 \mu \mathrm{m})$. QWP: quarter wave plate, WP: Wollaston prism, BPD: balance photodiode detector. (b) Pump laser spectrum with corresponding temporal pulse profile (c) measured with a scanning intensity autocorrelation. collimated manner. The $\mathrm{THz}$ pulse wavefront is thus expected to be free of high-order aberrations which should allow tight focusing. These expectations are corroborated by our experimental results. The temporal evolution of the electric field reconstructed by our EO sampling setup is shown in Fig. 2(a). The corresponding $\mathrm{THz}$ spectrum is plotted in Fig. 2(b) and covers a range of $0.1-5 \mathrm{THz}$. A pulse energy of up to $20 \mu \mathrm{J}$ has been measured with two independent and calibrated Golay cells (Tydex). ${ }^{20}$ Such high THz pulse energies are achieved since DAST is well phase-matched at mid-infrared wavelengths provided by our laser source $(1.2-1.5 \mu \mathrm{m}) .^{21}$ The maximum electric field strength of our $\mathrm{THz}$ source is calculated as $1.35 \mathrm{MV} / \mathrm{cm}$ at focus at a central frequency of $2.1 \mathrm{THz}$ and a bandwidth $\Delta v$ of $2 \mathrm{THz}$ (FWHM), considering the measured $\mathrm{THz}$ focus spot size $(0.7 \mathrm{~mm}$,

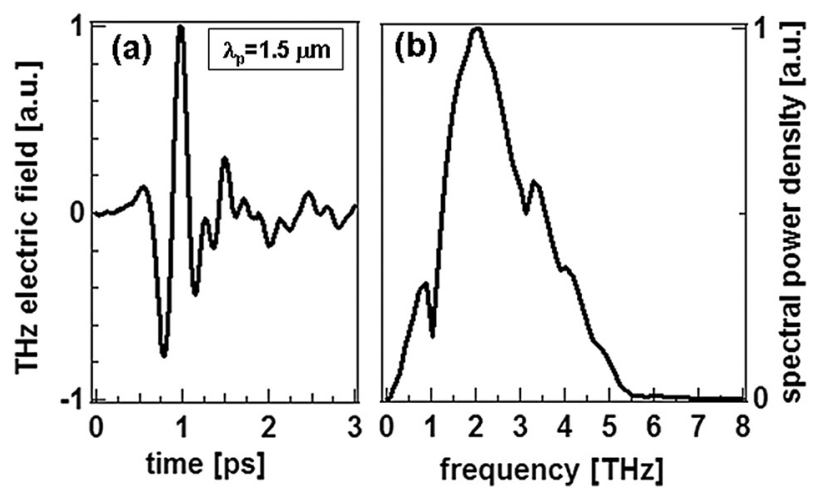

FIG. 2. (a) Single-cycle, phase-stabilized THz pulse from DAST pumped with $1.5 \mu \mathrm{m}$ radiation from EOS. The corresponding THz spectrum is plotted in (b). Field transients of up to $1.35 \mathrm{MV} / \mathrm{cm}$ are calculated by recording $\mathrm{THz}$ focus spot size and pulse energy with a Golay cell. reconstructed with the knife-edge technique), the pulse energy (Golay cell), and the pulse duration provided by the EOS trace. $^{22}$ The field strength reconstructed by measuring the maximum modulation signal of the balanced photo-detector $\left(\mathrm{I}_{1}-\mathrm{I}_{2}\right) /\left(\mathrm{I}_{1}+\mathrm{I}_{2}\right)=0.34( \pm 3 \%)$ on a CCD camera with signals $I_{1}$ and $I_{2}$ measured at the peak $\mathrm{THz}$ field indicates a somewhat lower field $(936 \mathrm{kV} / \mathrm{cm} \pm 10 \%)$ (Ref. 23) which is attributed to the measured CEP offset $\neq 0$ (see Fig. 2), to pulse distortions due to absorption and accumulated small uncertainties of filter transfer functions and of EOS crystal thickness. Considering a terahertz central frequency of 2.1 $\mathrm{THz}$ and a pump wavelength of $1.5 \mu \mathrm{m}$, a pump-to- $\mathrm{THz}$ energy conversion efficiency $\eta_{\mathrm{E}}=\left(E_{p} / E_{T H z}\right)$ of $2.2 \%$ is achieved, which corresponds to a photon conversion efficiency $\eta_{\mathrm{p}}=\left(N_{T H z} / N_{I R}\right)$ of $235 \%$.

The recorded $\mathrm{THz}$ spectrum shown in Figure 2(b) reveals an absorption line around 1.1 THz. This corresponds to a well-know absorption resonance in DAST originating from transverse optical (TO) phonon mode. ${ }^{24}$ Other spectral distortions caused by $\mathrm{THz}$ absorption in water vapor were significantly reduced by performing EOS measurement in dry air condition (relative humidity $<5 \%$ ). However, the residual spectral modulations still give rise to small temporal field oscillations posterior to the main pulse. In principle, the spectral modulation caused by TO phonon vibration could be reduced by cooling the crystal to very low temperature in order to damp those modes or by using other types of organic materials (e.g., OH1) with less pronounces phonon modes in this $\mathrm{THz}$ range. Other small temporal distortions in the wake of the main oscillation (Fig. 2(a)) are assigned to a ghost pulse originating from the internal reflection of the uncoated 
DAST. Higher fields could be realized by use of coated crystals, up-scaling the pump pulse energy, and by implementing a full control on the CEP offset. These implementations are currently under way.

Finally, we performed a parametric study of the $\mathrm{THz}$ radiation in DAST for different pump laser wavelengths. The results shown in Figure 3 indicate that the temporal and spectral pulse characteristics of the THz pulse (Figs. 3(a) and 3(b)) using a $1.2 \mu \mathrm{m}$ pump source are almost identical to the results shown in Figure $2\left(\lambda_{\mathrm{p}}=1.5 \mu \mathrm{m}\right)$ with laser parameters displayed in Table I. Similar THz characteristics are also measured for other pump wavelengths (Fig. 3(c)) where only little variations in the THz central frequency (circles), frequency bandwidth (triangles), or in the $\mathrm{THz}$ electric field strength (squares) are observed across the full spectral range of the pump laser. Laser-induced damage on the DAST surface is observed for intensities beyond $220 \mathrm{GW} / \mathrm{cm}^{2}$, which is comparable to previous results for DAST pumped with a Ti:sapphire laser. ${ }^{25}$

In conclusion, a powerful single-cycle $\mathrm{THz}$ source is presented emitting electric field amplitudes exceeding $1 \mathrm{MV} /$ $\mathrm{cm}$ electric and $0.3 \mathrm{~T}$ magnetic field strengths at a central frequency of $2.1 \mathrm{THz}$. The phase-locked THz pulses are efficiently generated by optical rectification in the organic DAST crystal, pumped by an intense infrared source. The high peak field and pulse energy of $\approx 20 \mu \mathrm{J}$ are ideally suited to drive extreme nonlinear phenomena in gases and solids with single-cycle electro-magnetic transients. These are one of the most intense phase-stable single-cycle $\mathrm{THz}$ pulses generated by laser-based table-top $\mathrm{THz}$ source in the $\mathrm{THz}$ frequency gap above $1 \mathrm{THz}$. The simplicity of the presented scheme allows the realization of highest field strengths without demanding pulse front shaping involved in other $\mathrm{THz}$ generation schemes. The source gives a prospect towards the
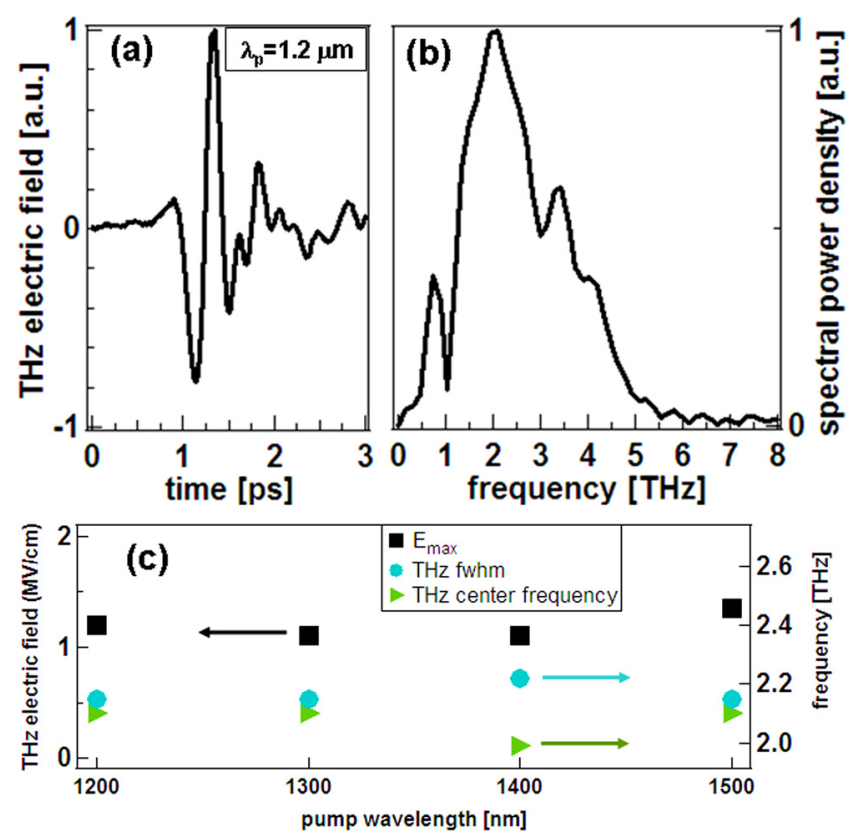

FIG. 3. (Color online) (a) Temporally (b) and spectrally reconstructed THz pulse for a DAST pumped with a $1.2 \mu \mathrm{m}$ laser. The results are similar to Fig. 2 indicating the $\mathrm{THz}$ generation process to be rather insensitive to the pump laser wavelength. (c) Calculated $\mathrm{THz}$ peak fields (squares), $\mathrm{THz}$ central frequency (circles), and generated THz bandwidth at FWHM (triangles) for different pump laser wavelengths.
TABLE I. Pump intensity and fluence on DAST for different pump wavelengths.

\begin{tabular}{lcccc}
\hline \hline$\lambda_{\text {pump }}(\mu \mathrm{m})$ & 1.2 & 1.3 & 1.4 & 1.5 \\
$\mathrm{E}_{\text {pump }}(\mu \mathrm{J})$ & 950 & 880 & 880 & 850 \\
$\tau_{\text {pump }}(\mathrm{fs})$ & 64 & 58 & 62 & 68 \\
$\mathrm{I}\left(\mathrm{GW} / \mathrm{cm}^{2}\right)$ & 173 & 177 & 166 & 145 \\
$\mathrm{~F}\left(\mathrm{~mJ} / \mathrm{cm}^{2}\right)$ & 11 & 10 & 10 & 10 \\
\hline \hline
\end{tabular}

realization of transients with even higher field strength by proper CEP offset control and by scaling up both the pump laser energy and the crystal size.

We acknowledge fruitful discussions with A. Trisorio and B. Patterson. We are grateful to V. Schlott for lending us the Golay cell and acknowledge support from R. Abela and H. Braun. C.P.H. acknowledges association to the NCCR-MUST network. This work has been supported by the Swiss National Foundation (PP00P2_128493/1 and 200021_122111/1) and SwissFEL.

${ }^{1}$ L. Razzari, F. H. Su, G. Sharma, F. Blanchard, A. Ayesheshim, H. C. Bandulet, R. Morandotti, J. C. Kieffer, T. Ozaki, M. Reid, and F. A. Hegmann, Phys. Rev. B 79, 193204 (2009).

${ }^{2}$ M. C. Hoffmann, J. Hebling, H. Y. Hwang, K. L. Yeh, and K. A. Nelson, Phys. Rev. B 79, 161201 (2009).

${ }^{3}$ B. E. Cole, J. B. Williams, B. T. King, M. S. Sherwin, and C. R. Stanley, Nature 410, 60 (2001).

${ }^{4}$ M. Tonouchi, Nature Photon. 1, 97 (2007).

${ }^{5}$ S. Leinss, T. Kampfrath, V. Vollkmann, M. Wolf, J. Steiner, M. Kira, S. Koch, A. Leitenstorfer, and R. Huber, Phys. Rev. Lett. 101, 246401 (2008).

${ }^{6}$ M. L. Cowan, B. D. Bruner, N. Huse, J. R. Dwyer, B. Chugh, E. T. J. Nibbering, T. Elsaesser, and R. J. D. Miller, Nature 434, 199 (2005).

${ }^{7}$ J. R. Danielson, Y.-S. Lee, J. P. Prineas, J. T. Steiner, M. Kira, and S. W. Koch, Phys. Rev. Lett. 99, 237401 (2007).

${ }^{8}$ F. Jungiger, A. Sell, O. Schubert, B. Mayer, D. Brida, M. Marangoni, G. Cerullo, A. Leitenstorfer, and R. Huber, Opt. Lett. 35, 2645 (2010).

${ }^{9}$ B. Ferguson and X. C. Zhang, Nature Mater. 1, 26 (2002).

${ }^{10}$ H. Hirori, A. Doi, F. Blanchard, and K. Tanaka, Appl. Phys. Lett. 98, 091106 (2011).

${ }^{11}$ T. Bartel, P. Gall, K. Reimann, M. Woerner, and T. Elsaesser, Opt. Lett. 30, 2805 (2005).

${ }^{12}$ For a review, see, e.g., A. S. Mueller, Rev. Accel. Sci. Technol. 3, 165 (2010) and references therein.

${ }^{13}$ X.-C. Zhang, X. F. Ma, Y. Jin, T.-M. Lu, E. P. Boden, P. D. Phelps, K. R. Stewart, and C. P. Yakymyshyn, Appl. Phys. Lett. 61, 308 (1992).

${ }^{14}$ P. Y. Han, M. Tani, F. Pan, and X.-C. Zhang, Opt. Lett. 25, 675 (2000).

${ }^{15}$ F. Brunner, O. Kwon, S. Kwon, M. Jazbinsek, A. Schneider, and P. Guenter, Opt. Express 16, 16496 (2008).

${ }^{16}$ A. Trisorio, P. M. Paul, F. Ple, C. Ruchert, C. Vicario, and C. P. Hauri, Opt. Express 19, 20128 (2011).

${ }^{17}$ H. Hirori, A. Doi, F. Blanchard, and K. Tanaka, Appl. Phys. Lett. 98, 091106 (2011).

${ }^{18}$ A. Baltuska, T. Fuji, and T. Kobayashi, Phys. Rev. Lett. 88, 133901 (2002).

${ }^{19}$ S. R. Marder, J. W. Perry, and W. P. Schaefer, Science 245, 626 (1989).

${ }^{20}$ The residual pump is blocked by a filter.

${ }^{21}$ A. Schneider, M. Neis, M. Stillhart, B. Ruiz, R. Kahn, and P. Gunter, J. Opt. Soc. Am. B 23, 1822 (2006).

${ }^{22} \mathrm{E}_{\mathrm{THz}}=\sqrt{0.5 \varepsilon_{T H z} /\left(c \varepsilon_{0} \tau_{T H z} \pi r_{T H z}^{2}\right)}$, with $\mathrm{E}_{\mathrm{THz}}$ electric field of $\mathrm{THz}, \mathrm{c}$ speed of light, $\varepsilon_{\mathrm{THz}} \mathrm{THz}$ pulse energy, $\mathrm{r}_{\mathrm{THz}}$ spot size radius, $\varepsilon_{0}$ electric permittivity of free space.

${ }^{23}$ Field reconstruction is performed according to $\sin ^{-1}(\Delta I / I)$ $=2 \pi n_{0}^{3} r_{41} t_{\text {GaP }} t_{S i} t_{T e} t_{\text {hm }} t_{\text {mirrors }} E_{T H z} L / \lambda_{0}$, with eo sampling coefficient $\mathrm{r}_{41}=0.88 \mathrm{pm} / \mathrm{V}$, crystal length $\mathrm{L}=95 \mu \mathrm{m}$, transmission coefficients $\mathrm{t}_{\mathrm{GaP}}=0.445, \mathrm{t}_{\mathrm{Si}}=0.65, \mathrm{t}_{\mathrm{Te}}=0.78, \mathrm{t}_{\mathrm{hm}}=0.81, \mathrm{t}_{\text {mirrors }}=0.96$ for $\mathrm{GaP}, \mathrm{Si}$, teflon, holey mirror, and the 4 mirrors used for beam transport.

${ }^{24}$ M. Walther, K. Jensby, S. R. Keding, H. Takahashi, and H. Ito, Opt. Lett. 25, 911 (2000).

${ }^{25}$ T. J. Carrig, G. Rodriguez, T. S. Clement, A. J. Taylor, and K. R. Stewart, Appl. Phys. Lett. 66, 121 (1995). 